\title{
BIPOLAR FUZZY SET THEORY APPLIED TO SUB-SEMIGROUPS WITH OPERATORS IN SEMIGROUPS
}

\author{
Mee Kwang Kang ${ }^{a, *}$ And JeOng Gi Kang ${ }^{\mathrm{b}}$
}

\begin{abstract}
Given a set $\Omega$ and the notion of bipolar valued fuzzy sets, the concept of a bipolar $\Omega$-fuzzy sub-semigroup in semigroups is introduced, and related properties are investigated. Using bipolar $\Omega$-fuzzy sub-semigroups, bipolar fuzzy sub-semigroups are constructed. Conversely, bipolar $\Omega$-fuzzy sub-semigroups are established by using bipolar fuzzy sub-semigroups. A characterizations of a bipolar $\Omega$-fuzzy sub-semigroup is provided, and normal bipolar $\Omega$-fuzzy sub-semigroups are discussed. How the homomorphic images and inverse images of bipolar $\Omega$-fuzzy sub-semigroups become bipolar $\Omega$-fuzzy sub-semigroups are considered.
\end{abstract}

\section{INTRODUCTION}

Fuzzy sets have been useful tools to bridge the gap between mathematical models and their empirical interpretations, and to deal with problems requiring the use of natural language(see $[1,6]$ ). A traditional fuzzy set is characterized by the membership function whose range is the unit interval $[0,1]$. There are several kinds of fuzzy set extensions in the fuzzy set theory, for example, intuitionistic fuzzy sets, intervalvalued fuzzy sets, vague sets etc. Lee [4] introduced an extension of fuzzy sets named bipolar-valued fuzzy sets to express the difference of the irrelevant elements from the contrary elements in fuzzy sets. Bipolar-valued fuzzy sets are an extension of fuzzy sets whose membership degree range is enlarged from the interval $[0,1]$ to $[-1,1]$. Bipolar-valued fuzzy sets have membership degrees that represent the degree of satisfaction to the property corresponding to a fuzzy set and its counter-property. Kim et al. [3] studied ideal theory of semigroups based on the bipolar valued fuzzy set

Received by the editors November 6, 2011. Revised January 26, 2012. Accepted Feb. 2, 2012. 2000 Mathematics Subject Classification. 20M12, 08A72.

Key words and phrases. bipolar fuzzy sub-semigroup, (normal) bipolar $\Omega$-fuzzy sub-semigroup, negative $s$-cut, positive $t$-cut.

${ }^{*}$ Corresponding author. 
theory. Hur et al. [2] discussed fuzzy sub-semigroups and fuzzy ideals with operators in semigroups.

In this paper, we deal with bipolar valued fuzzy theory with operators applied to sub-semigroups in semigroups. Using a set $\Omega$ and the notion of bipolar valued fuzzy sets, we introduce the concept of a bipolar $\Omega$-fuzzy sub-semigroup in semigroups, and investigate related properties. Using bipolar $\Omega$-fuzzy sub-semigroups, we construct bipolar fuzzy sub-semigroups, and conversely, we establish bipolar $\Omega$-fuzzy sub-semigroups by using bipolar fuzzy sub-semigroups. We provide a characterizations of a bipolar $\Omega$-fuzzy sub-semigroup, and discuss normal bipolar $\Omega$-fuzzy sub-semigroups. We consider how the homomorphic images and inverse images of bipolar $\Omega$-fuzzy sub-semigroups become bipolar $\Omega$-fuzzy sub-semigroups.

\section{Bipolar $\Omega$-FUZZY Sub-SEMigroups}

Let $S$ be the universe of discourse. A bipolar-valued fuzzy set $f$ in $S$ is an object having the form

$$
f=\left\{\left(x, f_{n}(x), f_{p}(x)\right) \mid x \in S\right\}
$$

where $f_{n}: S \rightarrow[-1,0]$ and $f_{p}: S \rightarrow[0,1]$ are mappings. The positive membership degree $f_{p}(x)$ denotes the satisfaction degree of an element $x$ to the property corresponding to a bipolar-valued fuzzy set $f=\left\{\left(x, f_{n}(x), f_{p}(x)\right) \mid x \in S\right\}$, and the negative membership degree $f_{n}(x)$ denotes the satisfaction degree of $x$ to some implicit counter-property of $f=\left\{\left(x, f_{n}(x), f_{p}(x)\right) \mid x \in S\right\}$. It is possible for an element $x$ to be $f_{p}(x) \neq 0$ and $f_{n}(x) \neq 0$ when the membership function of the property overlaps that of its counter-property over some portion of the domain (see [5] ). For the sake of simplicity, we shall use the symbol $f=\left(S ; f_{n}, f_{p}\right)$ for the bipolar-valued fuzzy set $f=\left\{\left(x, f_{n}(x), f_{p}(x)\right) \mid x \in S\right\}$, and use the notion of bipolar fuzzy sets instead of the notion of bipolar-valued fuzzy sets.

By a sub-semigroup of a semigroup $S$ we mean a nonempty subset $A$ of $S$ such that $A^{2} \subseteq A$.

A fuzzy set in $S$ is a function $\mu$ from $S$ into the unit interval $[0,1]$. A fuzzy set $\mu$ in $S$ is called a fuzzy sub-semigroup of $S$ if it satisfies

$$
(\forall x, y \in S)(\mu(x y) \geq \min \{\mu(x), \mu(y)\}) .
$$

For any family $\left\{a_{i} \mid i \in \Lambda\right\}$ of real numbers, we define

$$
\bigvee\left\{a_{i} \mid i \in \Lambda\right\}:= \begin{cases}\max \left\{a_{i} \mid i \in \Lambda\right\} & \text { if } \Lambda \text { is finite, } \\ \sup \left\{a_{i} \mid i \in \Lambda\right\} & \text { otherwise, }\end{cases}
$$




$$
\bigwedge\left\{a_{i} \mid i \in \Lambda\right\}:= \begin{cases}\min \left\{a_{i} \mid i \in \Lambda\right\} & \text { if } \Lambda \text { is finite, } \\ \inf \left\{a_{i} \mid i \in \Lambda\right\} & \text { otherwise. }\end{cases}
$$

A bipolar fuzzy set $f=\left(S ; f_{n}, f_{p}\right)$ in $S$ is called a bipolar fuzzy sub-semigroup of a semigroup $S$ (see [3]) if it satisfies the following condition:

$$
(\forall x, y \in S)\left(\begin{array}{l}
f_{n}(x y) \leq \bigvee\left\{f_{n}(x), f_{n}(y)\right\} \\
f_{p}(x y) \geq \bigwedge\left\{f_{p}(x), f_{p}(y)\right\}
\end{array}\right) .
$$

In what follows let $S$ and $\Omega$ denote a semigroup and a nonempty set, respectively, unless otherwise specified.

A bipolar $\Omega$-fuzzy set $F_{\Omega}$ in $S$ is defined to be an object having the form

$$
F_{\Omega}:=\left\{\left\langle(x, \alpha) ; f_{n}^{\Omega}(x, \alpha), f_{p}^{\Omega}(x, \alpha)\right\rangle \mid(x, \alpha) \in S \times \Omega\right\}
$$

where the function $f_{n}^{\Omega}: S \times \Omega \rightarrow[-1,0]$ and $f_{p}^{\Omega}: S \times \Omega \rightarrow[0,1]$.

We shall use the symbol $F_{\Omega}=\left\langle S \times \Omega ; f_{n}^{\Omega}, f_{p}^{\Omega}\right\rangle$ for the bipolar $\Omega$-fuzzy set

$$
F_{\Omega}:=\left\{\left\langle(x, \alpha) ; f_{n}^{\Omega}(x, \alpha), f_{p}^{\Omega}(x, \alpha)\right\rangle \mid(x, \alpha) \in S \times \Omega\right\} .
$$

Definition 2.1. A bipolar $\Omega$-fuzzy set $F_{\Omega}=\left\langle S \times \Omega ; f_{n}^{\Omega}, f_{p}^{\Omega}\right\rangle$ in $S$ is called a bipolar $\Omega$-fuzzy sub-semigroup of $S$ if it satisfies

$$
(\forall x, y \in S)(\forall \alpha \in \Omega)\left(\begin{array}{c}
f_{n}^{\Omega}(x y, \alpha) \leq \bigvee\left\{f_{n}^{\Omega}(x, \alpha), f_{n}^{\Omega}(y, \alpha)\right\}, \\
f_{p}^{\Omega}(x y, \alpha) \geq \bigwedge\left\{f_{p}^{\Omega}(x, \alpha), f_{p}^{\Omega}(y, \alpha)\right\}
\end{array}\right) .
$$

Example 2.2. Consider a semigroup $S=\{a, b\}$ with the following Cayley table:

$$
\begin{array}{l|ll} 
& a & b \\
\hline a & a & b \\
b & b & a
\end{array}
$$

Let $\Omega=\{1,2\}$ and let $F_{\Omega}=\left\langle S \times \Omega ; f_{n}^{\Omega}, f_{p}^{\Omega}\right\rangle$ be a bipolar $\Omega$-fuzzy set in $S$ defined by $F_{\Omega}=\{\langle(a, 1) ;-0.9,1\rangle,\langle(a, 2) ;-0.9,1\rangle,\langle(b, 1) ;-0.7,0.8\rangle,\langle(b, 2) ;-0.3,0.5\rangle\}$. It is easy to verify that $F_{\Omega}=\left\langle S \times \Omega ; f_{n}^{\Omega}, f_{p}^{\Omega}\right\rangle$ is a bipolar $\Omega$-fuzzy sub-semigroup of $S$.

Example 2.3. Let $S^{\Omega}:=\{u \mid u: \Omega \rightarrow S\}$. For any $u, v \in S^{\Omega}$, we define $(u v)(\alpha)=$ $u(\alpha) v(\alpha)$ for all $\alpha \in \Omega$. Then $S^{\Omega}$ is a semigroup. Let $f=\left(S ; f_{n}, f_{p}\right)$ be a bipolar fuzzy sub-semigroup of $S$ and let $\Phi_{\Omega}=\left\langle S^{\Omega} \times \Omega ; \Phi_{n}^{\Omega}, \Phi_{p}^{\Omega}\right\rangle$ where

$$
\Phi_{n}^{\Omega}: S^{\Omega} \times \Omega \rightarrow[-1,0],(u, \alpha) \mapsto f_{n}(u(\alpha))
$$

and

$$
\Phi_{p}^{\Omega}: S^{\Omega} \times \Omega \rightarrow[0,1], \quad(u, \alpha) \mapsto f_{p}(u(\alpha)) .
$$

Then $\Phi_{\Omega}=\left\langle S^{\Omega} \times \Omega ; \Phi_{n}^{\Omega}, \Phi_{p}^{\Omega}\right\rangle$ is a bipolar $\Omega$-fuzzy sub-semigroup of $S^{\Omega}$. 
Theorem 2.4. Let $\Omega$ be the set of all bipolar fuzzy sub-semigroups of $S$ and let $F_{\Omega}=\left\langle S \times \Omega ; f_{n}^{\Omega}, f_{p}^{\Omega}\right\rangle$ be a bipolar $\Omega$-fuzzy set in $S$ where $f_{n}^{\Omega}(x, f)=f_{n}(x)$ and $f_{p}^{\Omega}(x, f)=f_{p}(x)$ for all $x \in S$ and $f=\left(S ; f_{n}, f_{p}\right) \in \Omega$. Then $F_{\Omega}=\left\langle S \times \Omega ; f_{n}^{\Omega}, f_{p}^{\Omega}\right\rangle$ is a bipolar $\Omega$-fuzzy sub-semigroup of $S$.

Proof. Let $x, y \in S$ and $f=\left(S ; f_{n}, f_{p}\right) \in \Omega$. Then

$$
f_{n}^{\Omega}(x y, f)=f_{n}(x y) \leq \bigvee\left\{f_{n}(x), f_{n}(y)\right\}=\bigvee\left\{f_{n}^{\Omega}(x, f), f_{n}^{\Omega}(y, f)\right\}
$$

and

$$
f_{p}^{\Omega}(x y, f)=f_{p}(x y) \geq \bigwedge\left\{f_{p}(x), f_{p}(y)\right\}=\bigwedge\left\{f_{p}^{\Omega}(x, f), f_{p}^{\Omega}(y, f)\right\} .
$$

Hence $F_{\Omega}=\left\langle S \times \Omega ; f_{n}^{\Omega}, f_{p}^{\Omega}\right\rangle$ is a bipolar $\Omega$-fuzzy sub-semigroup of $S$.

Proposition 2.5. If $F_{\Omega}=\left\langle S \times \Omega ; f_{n}^{\Omega}, f_{p}^{\Omega}\right\rangle$ is a bipolar $\Omega$-fuzzy sub-semigroup of $S$ and $\alpha \in \Omega$, then a bipolar fuzzy set $f=\left(S ; f_{n}^{\alpha}, f_{p}^{\alpha}\right)$ where

$$
f_{n}^{\alpha}: S \rightarrow[-1,0], x \mapsto f_{n}^{\Omega}(x, \alpha)
$$

and

$$
f_{p}^{\alpha}: S \rightarrow[0,1], x \mapsto f_{p}^{\Omega}(x, \alpha)
$$

is a bipolar fuzzy sub-semigroup of $S$.

Proof. Let $x, y \in S$. Then

$$
f_{n}^{\alpha}(x y)=f_{n}^{\Omega}(x y, \alpha) \leq \bigvee\left\{f_{n}^{\Omega}(x, \alpha), f_{n}^{\Omega}(y, \alpha)\right\}=\bigvee\left\{f_{n}^{\alpha}(x), f_{n}^{\alpha}(y)\right\}
$$

and

$$
f_{p}^{\alpha}(x y)=f_{p}^{\Omega}(x y, \alpha) \geq \bigwedge\left\{f_{p}^{\Omega}(x, \alpha), f_{p}^{\Omega}(y, \alpha)\right\}=\bigwedge\left\{f_{p}^{\alpha}(x), f_{p}^{\alpha}(y)\right\} .
$$

This completes the proof.

Proposition 2.6. If $f=\left(S ; f_{n}^{\alpha}, f_{p}^{\alpha}\right), \alpha \in \Omega$, is a bipolar fuzzy sub-semigroup of $S$, then a bipolar $\Omega$-fuzzy set $F_{\Omega}=\left\langle S \times \Omega ; f_{n}^{\Omega}, f_{p}^{\Omega}\right\rangle$ where

$$
f_{n}^{\Omega}: S \times \Omega \rightarrow[-1,0], \quad(x, \alpha) \mapsto f_{n}^{\alpha}(x)
$$

and

$$
f_{p}^{\Omega}: S \times \Omega \rightarrow[0,1], \quad(x, \alpha) \mapsto f_{p}^{\alpha}(x)
$$

is a bipolar $\Omega$-fuzzy sub-semigroup of $S$.

Proof. For any $x, y \in S$, we have

$$
f_{n}^{\Omega}(x y, \alpha)=f_{n}^{\alpha}(x y) \leq \bigvee\left\{f_{n}^{\alpha}(x), f_{n}^{\alpha}(y)\right\}=\bigvee\left\{f_{n}^{\Omega}(x, \alpha), f_{n}^{\Omega}(y, \alpha)\right\}
$$


and

$$
f_{p}^{\Omega}(x y, \alpha)=f_{p}^{\alpha}(x y) \geq \bigwedge\left\{f_{p}^{\alpha}(x), f_{p}^{\alpha}(y)\right\}=\bigwedge\left\{f_{p}^{\Omega}(x, \alpha), f_{p}^{\Omega}(y, \alpha)\right\} .
$$

Hence $F_{\Omega}=\left\langle S \times \Omega ; f_{n}^{\Omega}, f_{p}^{\Omega}\right\rangle$ is a bipolar $\Omega$-fuzzy sub-semigroup of $S$.

Theorem 2.7. Let $\Phi_{\Omega}=\left\langle S^{\Omega} ; \Phi_{n}^{\Omega}, \Phi_{p}^{\Omega}\right\rangle$ be a bipolar fuzzy sub-semigroup of $S^{\Omega}$ and let $F_{\Omega}=\left\langle S \times \Omega ; f_{n}^{\Omega}, f_{p}^{\Omega}\right\rangle$ be a bipolar $\Omega$-fuzzy set in $S$ defined by

$$
\begin{aligned}
f_{n}^{\Omega}(x, \alpha) & :=\bigwedge\left\{\Phi_{n}^{\Omega}(u) \mid u \in S^{\Omega}, u(\alpha)=x\right\} \\
f_{p}^{\Omega}(x, \alpha) & :=\bigvee\left\{\Phi_{p}^{\Omega}(u) \mid u \in S^{\Omega}, u(\alpha)=x\right\}
\end{aligned}
$$

for all $x \in S$ and $\alpha \in \Omega$. Then $F_{\Omega}=\left\langle S \times \Omega ; f_{n}^{\Omega}, f_{p}^{\Omega}\right\rangle$ is a bipolar $\Omega$-fuzzy subsemigroup of $S$.

Proof. Let $x, y \in S$ and $\alpha \in \Omega$. Then

$$
\begin{aligned}
& f_{n}^{\Omega}(x y, \alpha)=\bigwedge\left\{\Phi_{n}^{\Omega}(u) \mid u \in S^{\Omega}, u(\alpha)=x y\right\} \\
& \leq \bigwedge\left\{\Phi_{n}^{\Omega}(u v) \mid u, v \in S^{\Omega}, u(\alpha)=x, v(\alpha)=y\right\} \\
& \leq \bigwedge\left\{\bigvee\left\{\Phi_{n}^{\Omega}(u), \Phi_{n}^{\Omega}(v)\right\} \mid u, v \in S^{\Omega}, u(\alpha)=x, v(\alpha)=y\right\} \\
& =\bigvee\left\{\bigwedge\left\{\Phi_{n}^{\Omega}(u) \mid u \in S^{\Omega}, u(\alpha)=x\right\}, \bigwedge\left\{\Phi_{n}^{\Omega}(v) \mid v \in S^{\Omega}, v(\alpha)=y\right\}\right\} \\
& =\bigvee\left\{f_{n}^{\Omega}(x, \alpha), f_{n}^{\Omega}(y, \alpha)\right\}
\end{aligned}
$$

and

$$
\begin{aligned}
& f_{p}^{\Omega}(x y, \alpha)=\bigvee\left\{\Phi_{p}^{\Omega}(u) \mid u \in S^{\Omega}, u(\alpha)=x y\right\} \\
& \geq \bigvee\left\{\Phi_{p}^{\Omega}(u v) \mid u, v \in S^{\Omega}, u(\alpha)=x, v(\alpha)=y\right\} \\
& \geq \bigvee\left\{\bigwedge\left\{\Phi_{p}^{\Omega}(u), \Phi_{p}^{\Omega}(v)\right\} \mid u, v \in S^{\Omega}, u(\alpha)=x, v(\alpha)=y\right\} \\
& =\bigwedge\left\{\bigvee\left\{\Phi_{p}^{\Omega}(u) \mid u \in S^{\Omega}, u(\alpha)=x\right\}, \bigvee\left\{\Phi_{p}^{\Omega}(v) \mid v \in S^{\Omega}, v(\alpha)=y\right\}\right\} \\
& =\bigwedge\left\{f_{p}^{\Omega}(x, \alpha), f_{p}^{\Omega}(y, \alpha)\right\} .
\end{aligned}
$$

Hence $F_{\Omega}=\left\langle S \times \Omega ; f_{n}^{\Omega}, f_{p}^{\Omega}\right\rangle$ is a bipolar $\Omega$-fuzzy sub-semigroup of $S$.

Example 2.8. Let $S=\{a, b\}$ be a semigroup in Example 2.2 and let $\Omega:=\{1,2\}$. Then $S^{\Omega}:=\{e, u, v, w\}$, where $e(1)=e(2)=v(1)=w(2)=a$ and $u(1)=u(2)=$ $v(2)=w(1)=b$, is a semigroup (in fact, a commutative group) under the following Cayley table (see [2]): 


\begin{tabular}{c|llll}
\hline & $e$ & $u$ & $v$ & $w$ \\
\hline$e$ & $e$ & $u$ & $v$ & $w$ \\
$u$ & $u$ & $e$ & $w$ & $v$ \\
$v$ & $v$ & $w$ & $e$ & $u$ \\
$w$ & $w$ & $v$ & $u$ & $e$ \\
\hline
\end{tabular}

Let $\Phi_{\Omega}=\left\langle S^{\Omega} ; \Phi_{n}^{\Omega}, \Phi_{p}^{\Omega}\right\rangle$ be a bipolar fuzzy set in $S^{\Omega}$ defined by

$$
\Phi_{\Omega}=\{\langle e ;-0.9,0.8\rangle,\langle u ;-0.3,0.2\rangle,\langle v ;-0.3,0.2\rangle,\langle w ;-0.5,0.7\rangle\} .
$$

Then $\Phi_{\Omega}=\left\langle S^{\Omega} ; \Phi_{n}^{\Omega}, \Phi_{p}^{\Omega}\right\rangle$ is a bipolar fuzzy sub-semigroup of $S^{\Omega}$. Thus we can obtain a bipolar $\Omega$-fuzzy sub-semigroup $F_{\Omega}=\left\langle S \times \Omega ; f_{n}^{\Omega}, f_{p}^{\Omega}\right\rangle$ of $S$ as follows:

$$
\begin{aligned}
& f_{n}^{\Omega}(a, 1)=\bigwedge\left\{\Phi_{n}^{\Omega}(\varnothing) \mid \varnothing \in S^{\Omega}, \varnothing(1)=a\right\}=\bigwedge\left\{\Phi_{n}^{\Omega}(e), \Phi_{n}^{\Omega}(v)\right\}=-0.9, \\
& f_{n}^{\Omega}(a, 2)=\bigwedge\left\{\Phi_{n}^{\Omega}(\varnothing) \mid \varnothing \in S^{\Omega}, \varnothing(2)=a\right\}=\bigwedge\left\{\Phi_{n}^{\Omega}(e), \Phi_{n}^{\Omega}(w)\right\}=-0.9 \\
& f_{n}^{\Omega}(b, 1)=\bigwedge\left\{\Phi_{n}^{\Omega}(\odot) \mid \varnothing \in S^{\Omega}, \varnothing(1)=b\right\}=\bigwedge\left\{\Phi_{n}^{\Omega}(u), \Phi_{n}^{\Omega}(w)\right\}=-0.5, \\
& f_{n}^{\Omega}(b, 2)=\bigwedge\left\{\Phi_{n}^{\Omega}(\varnothing) \mid \varnothing \in S^{\Omega}, \varnothing(2)=b\right\}=\bigwedge\left\{\Phi_{n}^{\Omega}(u), \Phi_{n}^{\Omega}(v)\right\}=-0.3, \\
& f_{p}^{\Omega}(a, 1)=\bigvee\left\{\Phi_{p}^{\Omega}(\mathbf{A}) \mid \mathbf{A} \in S^{\Omega}, \mathbf{\phi}(1)=a\right\}=\bigvee\left\{\Phi_{p}^{\Omega}(e), \Phi_{p}^{\Omega}(v)\right\}=0.8 \\
& f_{p}^{\Omega}(a, 2)=\bigvee\left\{\Phi_{p}^{\Omega}(\boldsymbol{A}) \mid \boldsymbol{\phi} \in S^{\Omega}, \boldsymbol{\phi}(2)=a\right\}=\bigvee\left\{\Phi_{p}^{\Omega}(e), \Phi_{p}^{\Omega}(w)\right\}=0.8,
\end{aligned}
$$

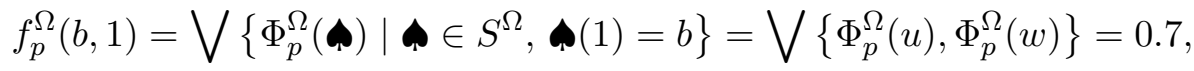

$$
\begin{aligned}
& f_{p}^{\Omega}(b, 2)=\bigvee\left\{\Phi_{p}^{\Omega}(\boldsymbol{\phi}) \mid \boldsymbol{\phi} \in S^{\Omega}, \boldsymbol{\phi}(2)=b\right\}=\bigvee\left\{\Phi_{p}^{\Omega}(u), \Phi_{p}^{\Omega}(v)\right\}=0.2
\end{aligned}
$$

Theorem 2.9. Let $F_{\Omega}=\left\langle S \times \Omega ; f_{n}^{\Omega}, f_{p}^{\Omega}\right\rangle$ be a bipolar $\Omega$-fuzzy sub-semigroup of $S$ and let $\Phi_{\Omega}=\left\langle S^{\Omega} ; \Phi_{n}^{\Omega}, \Phi_{p}^{\Omega}\right\rangle$ be a bipolar fuzzy set in $S^{\Omega}$ defined by

$$
\Phi_{n}^{\Omega}(u)=\bigvee\left\{f_{n}^{\Omega}(u(\alpha), \alpha) \mid \alpha \in \Omega\right\}
$$

and

$$
\Phi_{p}^{\Omega}(u)=\bigwedge\left\{f_{p}^{\Omega}(u(\alpha), \alpha) \mid \alpha \in \Omega\right\}
$$

for all $u \in S^{\Omega}$. Then $\Phi_{\Omega}=\left\langle S^{\Omega} ; \Phi_{n}^{\Omega}, \Phi_{p}^{\Omega}\right\rangle$ is a bipolar fuzzy sub-semigroup of $S^{\Omega}$. 
Proof. For any $u, v \in S^{\Omega}$, we have

$$
\begin{aligned}
\Phi_{n}^{\Omega}(u v) & =\bigvee\left\{f_{n}^{\Omega}((u v)(\alpha), \alpha) \mid \alpha \in \Omega\right\} \\
& =\bigvee\left\{f_{n}^{\Omega}(u(\alpha) v(\alpha), \alpha) \mid \alpha \in \Omega\right\} \\
& \leq \bigvee\left\{\bigvee\left\{f_{n}^{\Omega}(u(\alpha), \alpha), f_{n}^{\Omega}(v(\alpha), \alpha)\right\} \mid \alpha \in \Omega\right\} \\
& =\bigvee\left\{\bigvee\left\{f_{n}^{\Omega}(u(\alpha), \alpha) \mid \alpha \in \Omega\right\}, \bigvee\left\{f_{n}^{\Omega}(v(\alpha), \alpha) \mid \alpha \in \Omega\right\}\right\} \\
& =\bigvee\left\{\Phi_{n}^{\Omega}(u), \Phi_{n}^{\Omega}(v)\right\}
\end{aligned}
$$

and

$$
\begin{aligned}
\Phi_{p}^{\Omega}(u v) & =\bigwedge\left\{f_{p}^{\Omega}((u v)(\alpha), \alpha) \mid \alpha \in \Omega\right\} \\
& =\bigwedge\left\{f_{p}^{\Omega}(u(\alpha) v(\alpha), \alpha) \mid \alpha \in \Omega\right\} \\
& \geq \bigwedge\left\{\bigwedge\left\{f_{p}^{\Omega}(u(\alpha), \alpha), f_{p}^{\Omega}(v(\alpha), \alpha)\right\} \mid \alpha \in \Omega\right\} \\
& =\bigwedge\left\{\bigwedge\left\{f_{p}^{\Omega}(u(\alpha), \alpha) \mid \alpha \in \Omega\right\}, \bigwedge\left\{f_{p}^{\Omega}(v(\alpha), \alpha) \mid \alpha \in \Omega\right\}\right\} \\
& =\bigwedge\left\{\Phi_{p}^{\Omega}(u), \Phi_{p}^{\Omega}(v)\right\} .
\end{aligned}
$$

Thus $\Phi_{\Omega}=\left\langle S^{\Omega} ; \Phi_{n}^{\Omega}, \Phi_{p}^{\Omega}\right\rangle$ is a bipolar fuzzy sub-semigroup of $S^{\Omega}$.

Example 2.10. Let $F_{\Omega}=\left\langle S \times \Omega ; f_{n}^{\Omega}, f_{p}^{\Omega}\right\rangle$ be the bipolar $\Omega$-fuzzy sub-semigroup of $S$ in Example 2.2 and let $S^{\Omega}$ be the commutative group in Example 2.8. Then we can induce a bipolar fuzzy sub-semigroup $\Phi_{\Omega}=\left\langle S^{\Omega} ; \Phi_{n}^{\Omega}, \Phi_{p}^{\Omega}\right\rangle$ of $S^{\Omega}$ as follows:

$$
\begin{aligned}
\Phi_{n}^{\Omega}(e) & =\bigvee\left\{f_{n}^{\Omega}(e(\alpha), \alpha) \mid \alpha \in \Omega\right\}=\bigvee\left\{f_{n}^{\Omega}(e(1), 1), f_{n}^{\Omega}(e(2), 2)\right\} \\
& =\bigvee\left\{f_{n}^{\Omega}(a, 1), f_{n}^{\Omega}(a, 2)\right\}=-0.9, \\
\Phi_{n}^{\Omega}(u)= & \bigvee\left\{f_{n}^{\Omega}(u(\alpha), \alpha) \mid \alpha \in \Omega\right\}=\bigvee\left\{f_{n}^{\Omega}(u(1), 1), f_{n}^{\Omega}(u(2), 2)\right\} \\
& =\bigvee\left\{f_{n}^{\Omega}(b, 1), f_{n}^{\Omega}(b, 2)\right\}=-0.3, \\
\Phi_{n}^{\Omega}(v)= & \bigvee\left\{f_{n}^{\Omega}(v(\alpha), \alpha) \mid \alpha \in \Omega\right\}=\bigvee\left\{f_{n}^{\Omega}(v(1), 1), f_{n}^{\Omega}(v(2), 2)\right\} \\
& =\bigvee\left\{f_{n}^{\Omega}(a, 1), f_{n}^{\Omega}(b, 2)\right\}=-0.3, \\
\Phi_{n}^{\Omega}(w)= & \bigvee\left\{f_{n}^{\Omega}(w(\alpha), \alpha) \mid \alpha \in \Omega\right\}=\bigvee\left\{f_{n}^{\Omega}(w(1), 1), f_{n}^{\Omega}(w(2), 2)\right\} \\
& =\bigvee\left\{f_{n}^{\Omega}(b, 1), f_{n}^{\Omega}(a, 2)\right\}=-0.7, \\
\Phi_{p}^{\Omega}(e)= & \bigwedge\left\{f_{p}^{\Omega}(e(\alpha), \alpha) \mid \alpha \in \Omega\right\}=\bigwedge\left\{f_{p}^{\Omega}(e(1), 1), f_{p}^{\Omega}(e(2), 2)\right\} \\
& =\bigwedge\left\{f_{p}^{\Omega}(a, 1), f_{p}^{\Omega}(a, 2)\right\}=1, \\
\Phi_{p}^{\Omega}(u)= & \bigwedge\left\{f_{p}^{\Omega}(u(\alpha), \alpha) \mid \alpha \in \Omega\right\}=\bigwedge\left\{f_{p}^{\Omega}(u(1), 1), f_{p}^{\Omega}(u(2), 2)\right\} \\
& =\bigwedge\left\{f_{p}^{\Omega}(b, 1), f_{p}^{\Omega}(b, 2)\right\}=0.5, \\
\Phi_{p}^{\Omega}(v)= & \bigwedge\left\{f_{p}^{\Omega}(v(\alpha), \alpha) \mid \alpha \in \Omega\right\}=\bigwedge\left\{f_{p}^{\Omega}(v(1), 1), f_{p}^{\Omega}(v(2), 2)\right\} \\
= & \bigwedge\left\{f_{p}^{\Omega}(a, 1), f_{p}^{\Omega}(b, 2)\right\}=0.5,
\end{aligned}
$$




$$
\begin{aligned}
\Phi_{p}^{\Omega}(w) & =\bigwedge\left\{f_{p}^{\Omega}(w(\alpha), \alpha) \mid \alpha \in \Omega\right\}=\bigwedge\left\{f_{p}^{\Omega}(w(1), 1), f_{p}^{\Omega}(w(2), 2)\right\} \\
& =\bigwedge\left\{f_{p}^{\Omega}(b, 1), f_{p}^{\Omega}(a, 2)\right\}=0.8
\end{aligned}
$$

For a bipolar $\Omega$-fuzzy set $F_{\Omega}=\left\langle S \times \Omega ; f_{n}^{\Omega}, f_{p}^{\Omega}\right\rangle$ in $S$ and $(s, t) \in[-1,0] \times[0,1]$, we define

$$
\begin{aligned}
& N\left(F_{\Omega} ; s\right)=\left\{x \in S \mid f_{n}^{\Omega}(x, \alpha) \leq s, \forall \alpha \in \Omega\right\}, \\
& P\left(F_{\Omega} ; t\right)=\left\{x \in S \mid f_{p}^{\Omega}(x, \alpha) \geq t, \forall \alpha \in \Omega\right\}
\end{aligned}
$$

which are called the negative $s$-cut of $F_{\Omega}=\left\langle S \times \Omega ; f_{n}^{\Omega}, f_{p}^{\Omega}\right\rangle$ and the positive $t$-cut of $F_{\Omega}=\left\langle S \times \Omega ; f_{n}^{\Omega}, f_{p}^{\Omega}\right\rangle$, respectively. The set

$$
C\left(F_{\Omega} ;(s, t)\right):=N\left(F_{\Omega} ; s\right) \cap P\left(F_{\Omega} ; t\right)
$$

is called the $(s, t)$-cut of $F_{\Omega}=\left\langle S \times \Omega ; f_{n}^{\Omega}, f_{p}^{\Omega}\right\rangle$. For every $k \in[0,1]$, if $(s, t)=(-k, k)$ then the set

$$
C\left(F_{\Omega} ; k\right):=N\left(F_{\Omega} ;-k\right) \cap P\left(F_{\Omega} ; k\right)
$$

is called the $k$-cut of $F_{\Omega}=\left\langle S \times \Omega ; f_{n}^{\Omega}, f_{p}^{\Omega}\right\rangle$.

Theorem 2.11. If a bipolar $\Omega$-fuzzy set $F_{\Omega}=\left\langle S \times \Omega ; f_{n}^{\Omega}, f_{p}^{\Omega}\right\rangle$ in $S$ is a bipolar $\Omega$-fuzzy sub-semigroup of $S$, then the following assertions are valid:

(1) $(\forall s \in[-1,0])\left(N\left(F_{\Omega} ; s\right) \neq \emptyset \Rightarrow N\left(F_{\Omega} ; s\right)\right.$ is a sub-semigroup of $\left.S\right)$.

(2) $(\forall t \in[0,1])\left(P\left(F_{\Omega} ; t\right) \neq \emptyset \Rightarrow P\left(F_{\Omega} ; t\right)\right.$ is a sub-semigroup of $\left.S\right)$.

Proof. Let $s \in[-1,0]$ be such that $N\left(F_{\Omega} ; s\right) \neq \emptyset$. If $x, y \in N\left(F_{\Omega} ; s\right)$, then $f_{n}^{\Omega}(x, \alpha) \leq$ $s$ and $f_{n}^{\Omega}(y, \alpha) \leq s$ for all $\alpha \in \Omega$. It follows that

$$
f_{n}^{\Omega}(x y, \alpha) \leq \bigvee\left\{f_{n}^{\Omega}(x, \alpha), f_{n}^{\Omega}(y, \alpha)\right\} \leq s
$$

so that $x y \in N\left(F_{\Omega} ; s\right)$. Hence $N\left(F_{\Omega} ; s\right)$ is a sub-semigroup of $S$. Now, let $t \in[0,1]$ be such that $P\left(F_{\Omega} ; t\right) \neq \emptyset$. If $x, y \in P\left(F_{\Omega} ; t\right)$, then $f_{p}^{\Omega}(x, \alpha) \geq t$ and $f_{p}^{\Omega}(y, \alpha) \geq t$ for all $\alpha \in \Omega$, and so

$$
f_{p}^{\Omega}(x y, \alpha) \geq \bigwedge\left\{f_{p}^{\Omega}(x, \alpha), f_{p}^{\Omega}(y, \alpha)\right\} \geq t .
$$

Therefore $P\left(F_{\Omega} ; t\right)$ is a sub-semigroup of $S$.

Corollary 2.12. If a bipolar $\Omega$-fuzzy set $F_{\Omega}=\left\langle S \times \Omega ; f_{n}^{\Omega}, f_{p}^{\Omega}\right\rangle$ in $S$ is a bipolar $\Omega$ fuzzy sub-semigroup of $S$, then the $k$-cut of $F_{\Omega}=\left\langle S \times \Omega ; f_{n}^{\Omega}, f_{p}^{\Omega}\right\rangle$ is a sub-semigroup of $S$ for all $k \in[0,1]$.

We now consider the converse of Theorem 2.11. 
Theorem 2.13. Let $F_{\Omega}=\left\langle S \times \Omega ; f_{n}^{\Omega}, f_{p}^{\Omega}\right\rangle$ be a bipolar $\Omega$-fuzzy set in $S$ satisfying two conditions (1) and (2) in Theorem 2.11. Then $F_{\Omega}=\left\langle S \times \Omega ; f_{n}^{\Omega}, f_{p}^{\Omega}\right\rangle$ is a bipolar $\Omega$-fuzzy sub-semigroup of $S$.

Proof. Assume that $F_{\Omega}=\left\langle S \times \Omega ; f_{n}^{\Omega}, f_{p}^{\Omega}\right\rangle$ is not a bipolar $\Omega$-fuzzy sub-semigroup of $S$. Then the condition (2.2) is false, that is, there exist $a, b \in S$ and $\alpha \in \Omega$ such that $f_{n}^{\Omega}(a b, \alpha)>\bigvee\left\{f_{n}^{\Omega}(a, \alpha), f_{n}^{\Omega}(b, \alpha)\right\}$ or $f_{p}^{\Omega}(a b, \alpha)<\bigwedge\left\{f_{p}^{\Omega}(a, \alpha), f_{p}^{\Omega}(b, \alpha)\right\}$. If $f_{n}^{\Omega}(a b, \alpha)>\bigvee\left\{f_{n}^{\Omega}(a, \alpha), f_{n}^{\Omega}(b, \alpha)\right\}$, then

$$
f_{n}^{\Omega}(a b, \alpha)>s_{\alpha} \geq \bigvee\left\{f_{n}^{\Omega}(a, \alpha), f_{n}^{\Omega}(b, \alpha)\right\}
$$

for some $s_{\alpha} \in[-1,0]$. It follows that $a, b \in N\left(F_{\Omega} ; s_{\alpha}\right)$ but $a b \notin N\left(F_{\Omega} ; s_{\alpha}\right)$ which is a contradiction. Therefore $f_{n}^{\Omega}(x y, \alpha) \leq \bigvee\left\{f_{n}^{\Omega}(x, \alpha), f_{n}^{\Omega}(y, \alpha)\right\}$ for all $x, y \in S$ and $\alpha \in \Omega$. Now, if $f_{p}^{\Omega}(a b, \alpha)<\bigwedge\left\{f_{p}^{\Omega}(a, \alpha), f_{p}^{\Omega}(b, \alpha)\right\}$, then

$$
f_{p}^{\Omega}(a b, \alpha)<t_{\alpha} \leq \bigwedge\left\{f_{p}^{\Omega}(a, \alpha), f_{p}^{\Omega}(b, \alpha)\right\}
$$

and so $a, b \in P\left(F_{\Omega} ; t_{\alpha}\right)$ but $a b \notin P\left(F_{\Omega} ; t_{\alpha}\right)$. Thus $P\left(F_{\Omega} ; t_{\alpha}\right)$ is not a sub-semigroup of $S$, which is a contradiction. Consequently, $F_{\Omega}=\left\langle S \times \Omega ; f_{n}^{\Omega}, f_{p}^{\Omega}\right\rangle$ is a bipolar $\Omega$-fuzzy sub-semigroup of $S$.

Definition 2.14. A bipolar $\Omega$-fuzzy sub-semigroup $F_{\Omega}=\left\langle S \times \Omega ; f_{n}^{\Omega}, f_{p}^{\Omega}\right\rangle$ of $S$ is said to be normal if it satisfies:

$$
(\forall \alpha \in \Omega)(\exists x, y \in S)\left(f_{n}^{\Omega}(x, \alpha)=-1 \text { and } f_{p}^{\Omega}(y, \alpha)=1\right) .
$$

Example 2.15. Consider a semigroup $S^{\Omega}:=\{e, u, v, w\}$ which is described in Example 2.8. Let $\Phi_{\Omega}=\left\langle S^{\Omega} ; \Phi_{n}^{\Omega}, \Phi_{p}^{\Omega}\right\rangle$ be a bipolar fuzzy set in $S^{\Omega}$ defined by

$$
\Phi_{\Omega}=\{\langle e ;-1,1\rangle,\langle u ;-0.3,0.2\rangle,\langle v ;-0.3,0.2\rangle,\langle w ;-0.5,0.7\rangle\} .
$$

Then $\Phi_{\Omega}=\left\langle S^{\Omega} ; \Phi_{n}^{\Omega}, \Phi_{p}^{\Omega}\right\rangle$ is a bipolar fuzzy sub-semigroup of $S^{\Omega}$, which induces a bipolar fuzzy sub-semigroup $\Phi_{\Omega}=\left\langle S^{\Omega} ; \Phi_{n}^{\Omega}, \Phi_{p}^{\Omega}\right\rangle$ of $S^{\Omega}$ where

$$
\begin{aligned}
& f_{n}^{\Omega}(a, 1)=f_{n}^{\Omega}(a, 2)=-1, f_{n}^{\Omega}(b, 1)=-0.5, f_{n}^{\Omega}(b, 2)=-0.3, \\
& f_{p}^{\Omega}(a, 1)=f_{p}^{\Omega}(a, 2)=1, f_{p}^{\Omega}(b, 1)=0.7, f_{p}^{\Omega}(b, 2)=0.2 .
\end{aligned}
$$

Thus $F_{\Omega}=\left\langle S \times \Omega ; f_{n}^{\Omega}, f_{p}^{\Omega}\right\rangle$ is a normal bipolar $\Omega$-fuzzy sub-semigroup of $S$.

Let $\Sigma$ denote the set of all normal bipolar $\Omega$-fuzzy sub-semigroups of $S$. Denote by $\theta$ the special element of $S$ such that

$$
(\forall \alpha \in \Omega)\left(f_{n}^{\Omega}(\theta, \alpha)=\bigwedge_{x \in S} f_{n}^{\Omega}(x, \alpha) \text { and } f_{p}^{\Omega}(\theta, \alpha)=\bigvee_{x \in S} f_{p}^{\Omega}(x, \alpha)\right) .
$$


Clearly, if $F_{\Omega}=\left\langle S \times \Omega ; f_{n}^{\Omega}, f_{p}^{\Omega}\right\rangle$ is a normal bipolar $\Omega$-fuzzy sub-semigroup of $S$, then $f_{n}^{\Omega}(\theta, \alpha)=-1$ and $f_{p}^{\Omega}(\theta, \alpha)=1$ for all $\alpha \in \Omega$.

We consider a method for making a normal bipolar $\Omega$-fuzzy sub-semigroup from a given bipolar $\Omega$-fuzzy sub-semigroup.

Theorem 2.16. Let $F_{\Omega}=\left\langle S \times \Omega ; f_{n}^{\Omega}, f_{p}^{\Omega}\right\rangle$ be a bipolar $\Omega$-fuzzy sub-semigroup of $S$. Let $\overline{F_{\Omega}}=\left\langle S \times \Omega ; \overline{f_{n}^{\Omega}}, \overline{f_{p}^{\Omega}}\right\rangle$ be a bipolar fuzzy set in $S$ defined by

$$
\overline{f_{n}^{\Omega}}(x, \alpha)=f_{n}^{\Omega}(x, \alpha)-1+f_{n}^{\Omega}(\theta, \alpha) \text { and } \overline{f_{p}^{\Omega}}(x, \alpha)=f_{p}^{\Omega}(x, \alpha)+1-f_{p}^{\Omega}(\theta, \alpha)
$$

for all $\alpha \in \Omega$ and $x \in S$. Then $\overline{F_{\Omega}}=\left\langle S \times \Omega ; \overline{f_{n}^{\Omega}}, \overline{f_{p}^{\Omega}}\right\rangle$ is a normal bipolar $\Omega$-fuzzy sub-semigroup of $S$.

Proof. For all $x, y \in S$ and $\alpha \in \Omega$, we have

$$
\begin{aligned}
\overline{f_{n}^{\Omega}}(x y, \alpha) & =f_{n}^{\Omega}(x y, \alpha)-1+f_{n}^{\Omega}(\theta, \alpha) \\
& \leq \bigvee\left\{f_{n}^{\Omega}(x, \alpha), f_{n}^{\Omega}(y, \alpha)\right\}-1+f_{n}^{\Omega}(\theta, \alpha) \\
& =\bigvee\left\{f_{n}^{\Omega}(x, \alpha)-1+f_{n}^{\Omega}(\theta, \alpha), f_{n}^{\Omega}(y, \alpha)-1+f_{n}^{\Omega}(\theta, \alpha)\right\} \\
& =\bigvee\left\{\overline{f_{n}^{\Omega}}(x, \alpha), \overline{f_{n}^{\Omega}}(y, \alpha)\right\}
\end{aligned}
$$

and

$$
\begin{aligned}
\overline{f_{p}^{\Omega}}(x y, \alpha) & =f_{p}^{\Omega}(x y, \alpha)+1-f_{p}^{\Omega}(\theta, \alpha) \\
& \geq \bigwedge\left\{f_{p}^{\Omega}(x, \alpha), f_{p}^{\Omega}(y, \alpha)\right\}+1-f_{p}^{\Omega}(\theta, \alpha) \\
& =\bigwedge\left\{f_{p}^{\Omega}(x, \alpha)+1-f_{p}^{\Omega}(\theta, \alpha), f_{p}^{\Omega}(y, \alpha)+1-f_{p}^{\Omega}(\theta, \alpha)\right\} \\
& =\bigwedge\left\{\overline{f_{p}^{\Omega}}(x, \alpha), \overline{f_{p}^{\Omega}}(y, \alpha)\right\} .
\end{aligned}
$$

Clearly, $\overline{f_{n}^{\Omega}}(\theta, \alpha)=-1$ and $\overline{f_{p}^{\Omega}}(\theta, \alpha)=1$. Thus $F_{\Omega}=\left\langle S \times \Omega ; f_{n}^{\Omega}, f_{p}^{\Omega}\right\rangle$ is a normal bipolar $\Omega$-fuzzy sub-semigroup of $S$.

Definition 2.17. Let $\varphi: S \rightarrow T$ be a homomorphism of semigroups and let $G_{\Omega}=\left\langle T \times \Omega ; g_{n}^{\Omega}, g_{p}^{\Omega}\right\rangle$ be a bipolar $\Omega$-fuzzy set in $T$. Then the inverse image of $G_{\Omega}=\left\langle T \times \Omega ; g_{n}^{\Omega}, g_{p}^{\Omega}\right\rangle$, denoted by $\varphi^{-1}\left[G_{\Omega}\right]=\left\langle T \times \Omega ; \varphi^{-1}\left(g_{n}^{\Omega}\right), \varphi^{-1}\left(g_{p}^{\Omega}\right)\right\rangle$ is the bipolar $\Omega$-fuzzy set in $S$ given by $\varphi^{-1}\left(g_{n}^{\Omega}\right)(x, \alpha)=g_{n}^{\Omega}(\varphi(x), \alpha)$ and $\varphi^{-1}\left(g_{p}^{\Omega}\right)(x, \alpha)=$ $g_{p}^{\Omega}(\varphi(x), \alpha)$ for all $x \in S$ and $\alpha \in \Omega$. Conversely, let $F_{\Omega}=\left\langle S \times \Omega ; f_{n}^{\Omega}, f_{p}^{\Omega}\right\rangle$ be a bipolar $\Omega$-fuzzy set in $S$. The image of $F_{\Omega}=\left\langle S \times \Omega ; f_{n}^{\Omega}, f_{p}^{\Omega}\right\rangle$, written as $\varphi\left[F_{\Omega}\right]=\left\langle T \times \Omega ; \varphi\left(f_{n}^{\Omega}\right), \varphi\left(f_{p}^{\Omega}\right)\right\rangle$, is a bipolar $\Omega$-fuzzy set in $T$ defined by

$$
\varphi\left(f_{n}^{\Omega}\right)(y, \alpha)= \begin{cases}\bigwedge_{z \in \varphi^{-1}(y)} f_{n}^{\Omega}(z, \alpha) & \text { if } \varphi^{-1}(y) \neq \emptyset, \\ 0 & \text { otherwise }\end{cases}
$$




$$
\varphi\left(f_{p}^{\Omega}\right)(y, \alpha)= \begin{cases}\bigvee_{z \in \varphi^{-1}(y)} f_{p}^{\Omega}(z, \alpha) & \text { if } \varphi^{-1}(y) \neq \emptyset, \\ 0 & \text { otherwise }\end{cases}
$$

for all $y \in T$ and $\alpha \in \Omega$, where $\varphi^{-1}(y)=\{x \mid \varphi(x)=y\}$.

Theorem 2.18. Let $\varphi: S \rightarrow T$ be a homomorphism of semigroups and let $G_{\Omega}=$ $\left\langle T \times \Omega ; g_{n}^{\Omega}, g_{p}^{\Omega}\right\rangle$ be a bipolar $\Omega$-fuzzy sub-semigroup of $T$. Then its inverse image $\varphi^{-1}\left[G_{\Omega}\right]=\left\langle T \times \Omega ; \varphi^{-1}\left(g_{n}^{\Omega}\right), \varphi^{-1}\left(g_{p}^{\Omega}\right)\right\rangle$ is a bipolar $\Omega$-fuzzy sub-semigroup of $S$.

Proof. Let $x, y \in S$ and $\alpha \in \Omega$. Then

$$
\begin{aligned}
\varphi^{-1}\left(g_{n}^{\Omega}\right)(x y, \alpha) & =g_{n}^{\Omega}(\varphi(x y), \alpha)=g_{n}^{\Omega}(\varphi(x) \varphi(x), \alpha) \\
& \leq \bigvee\left\{g_{n}^{\Omega}(\varphi(x), \alpha), g_{n}^{\Omega}(\varphi(y), \alpha)\right\} \\
& =\bigvee\left\{\varphi^{-1}\left(g_{n}^{\Omega}\right)(x, \alpha), \varphi^{-1}\left(g_{n}^{\Omega}\right)(y, \alpha)\right\}
\end{aligned}
$$

and

$$
\begin{aligned}
\varphi^{-1}\left(g_{p}^{\Omega}\right)(x y, \alpha) & =g_{p}^{\Omega}(\varphi(x y), \alpha)=g_{p}^{\Omega}(\varphi(x) \varphi(x), \alpha) \\
& \geq \bigwedge\left\{g_{p}^{\Omega}(\varphi(x), \alpha), g_{p}^{\Omega}(\varphi(y), \alpha)\right\} \\
& =\bigwedge\left\{\varphi^{-1}\left(g_{p}^{\Omega}\right)(x, \alpha), \varphi^{-1}\left(g_{p}^{\Omega}\right)(y, \alpha)\right\} .
\end{aligned}
$$

Hence $\varphi^{-1}\left[G_{\Omega}\right]=\left\langle T \times \Omega ; \varphi^{-1}\left(g_{n}^{\Omega}\right), \varphi^{-1}\left(g_{p}^{\Omega}\right)\right\rangle$ is a bipolar $\Omega$-fuzzy sub-semigroup of $S$.

Theorem 2.19. Let $\varphi: S \rightarrow T$ be a homomorphism between semigroups $S$ and $T$. If $F_{\Omega}=\left\langle S \times \Omega ; f_{n}^{\Omega}, f_{p}^{\Omega}\right\rangle$ is a bipolar $\Omega$-fuzzy sub-semigroup of $S$, then the image $\varphi\left[F_{\Omega}\right]=\left\langle T \times \Omega ; \varphi\left(f_{n}^{\Omega}\right), \varphi\left(f_{p}^{\Omega}\right)\right\rangle$ is a bipolar $\Omega$-fuzzy sub-semigroup of $T$.

Proof. We first prove that

$$
\varphi^{-1}\left(y_{1}\right) \varphi^{-1}\left(y_{2}\right) \subseteq \varphi^{-1}\left(y_{1} y_{2}\right)
$$

for all $y_{1}, y_{2} \in T$. For, if $x \in \varphi^{-1}\left(y_{1}\right) \varphi^{-1}\left(y_{2}\right)$, then $x=x_{1} x_{2}$ for some $x_{1} \in \varphi^{-1}\left(y_{1}\right)$ and $x_{2} \in \varphi^{-1}\left(y_{2}\right)$. Since $\varphi$ is a homomorphism, it follows that

$$
\varphi(x)=\varphi\left(x_{1} x_{2}\right)=\varphi\left(x_{1}\right) \varphi\left(x_{2}\right)=y_{1} y_{2}
$$

so that $x \in \varphi^{-1}\left(y_{1} y_{2}\right)$. Hence (2.5) holds. Now let $y_{1}, y_{2} \in T$ and $\alpha \in \Omega$. Assume that $y_{1} y_{2} \notin \operatorname{Im}(\varphi)$. Then $\varphi\left(f_{n}^{\Omega}\right)\left(y_{1} y_{2}, \alpha\right)=0=\varphi\left(f_{p}^{\Omega}\right)\left(y_{1} y_{2}, \alpha\right)$. But if $y_{1} y_{2} \notin$ $\operatorname{Im}(\varphi)$, i.e., $\varphi^{-1}\left(y_{1} y_{2}\right)=\emptyset$, then $\varphi^{-1}\left(y_{1}\right)=\emptyset$ or $\varphi^{-1}\left(y_{2}\right)=\emptyset$ by (2.5). Thus $\varphi\left(f_{n}^{\Omega}\right)\left(y_{1}, \alpha\right)=0=\varphi\left(f_{p}^{\Omega}\right)\left(y_{1}, \alpha\right)$ or $\varphi\left(f_{n}^{\Omega}\right)\left(y_{2}, \alpha\right)=0=\varphi\left(f_{p}^{\Omega}\right)\left(y_{2}, \alpha\right)$, and so

$$
\begin{aligned}
& \varphi\left(f_{n}^{\Omega}\right)\left(y_{1} y_{2}, \alpha\right)=0=\bigwedge\left\{\varphi\left(f_{n}^{\Omega}\right)\left(y_{1}, \alpha\right), \varphi\left(f_{n}^{\Omega}\right)\left(y_{2}, \alpha\right)\right\}, \\
& \varphi\left(f_{p}^{\Omega}\right)\left(y_{1} y_{2}, \alpha\right)=0=\bigvee\left\{\varphi\left(f_{p}^{\Omega}\right)\left(y_{1}, \alpha\right), \varphi\left(f_{p}^{\Omega}\right)\left(y_{2}, \alpha\right)\right\} .
\end{aligned}
$$


Suppose that $\varphi^{-1}\left(y_{1} y_{2}\right) \neq \emptyset$. Then we should consider two cases as follows:

(i) $\varphi^{-1}\left(y_{1}\right)=\emptyset$ or $\varphi^{-1}\left(y_{2}\right)=\emptyset$,

(ii) $\varphi^{-1}\left(y_{1}\right) \neq \emptyset$ and $\varphi^{-1}\left(y_{2}\right) \neq \emptyset$.

For the first case, we have $\varphi\left(f_{n}^{\Omega}\right)\left(y_{1}, \alpha\right)=0=\varphi\left(f_{p}^{\Omega}\right)\left(y_{1}, \alpha\right)$ or $\varphi\left(f_{n}^{\Omega}\right)\left(y_{2}, \alpha\right)=0=$ $\varphi\left(f_{p}^{\Omega}\right)\left(y_{2}, \alpha\right)$. Hence

$$
\varphi\left(f_{n}^{\Omega}\right)\left(y_{1} y_{2}, \alpha\right) \leq \bigwedge\left\{\varphi\left(f_{n}^{\Omega}\right)\left(y_{1}, \alpha\right), \varphi\left(f_{n}^{\Omega}\right)\left(y_{2}, \alpha\right)\right\}
$$

and

$$
\varphi\left(f_{p}^{\Omega}\right)\left(y_{1} y_{2}, \alpha\right) \geq \bigvee\left\{\varphi\left(f_{p}^{\Omega}\right)\left(y_{1}, \alpha\right), \varphi\left(f_{p}^{\Omega}\right)\left(y_{2}, \alpha\right)\right\} .
$$

Case (ii) implies that

$$
\begin{aligned}
& \varphi\left(f_{n}^{\Omega}\right)\left(y_{1} y_{2}, \alpha\right)=\bigwedge_{z \in \varphi^{-1}\left(y_{1} y_{2}\right)} f_{n}^{\Omega}(z, \alpha) \leq \bigwedge_{z \in \varphi^{-1}\left(y_{1}\right) \varphi^{-1}\left(y_{2}\right)} f_{n}^{\Omega}(z, \alpha) \\
& =\bigwedge_{\substack{x_{1} \in \varphi^{-1}\left(y_{1}\right) \\
x_{2} \in \varphi^{-1}\left(y_{2}\right)}} f_{n}^{\Omega}\left(x_{1} x_{2}, \alpha\right) \leq \bigwedge_{\substack{x_{1} \in \varphi^{-1}\left(y_{1}\right) \\
x_{2} \in \varphi^{-1}\left(y_{2}\right)}} \bigvee\left\{f_{n}^{\Omega}\left(x_{1}, \alpha\right), f_{n}^{\Omega}\left(x_{2}, \alpha\right)\right\} \\
& =\bigvee\left\{\bigwedge_{x_{1} \in \varphi^{-1}\left(y_{1}\right)} f_{n}^{\Omega}\left(x_{1}, \alpha\right), \bigwedge_{x_{2} \in \varphi^{-1}\left(y_{2}\right)} f_{n}^{\Omega}\left(x_{2}, \alpha\right)\right\} \\
& =\bigvee\left\{\varphi\left(f_{n}^{\Omega}\right)\left(y_{1}, \alpha\right), \varphi\left(f_{n}^{\Omega}\right)\left(y_{2}, \alpha\right)\right\}
\end{aligned}
$$

and

$$
\begin{aligned}
& \varphi\left(f_{p}^{\Omega}\right)\left(y_{1} y_{2}, \alpha\right)=\bigvee_{z \in \varphi^{-1}\left(y_{1} y_{2}\right)} f_{p}^{\Omega}(z, \alpha) \geq \bigvee_{z \in \varphi^{-1}\left(y_{1}\right) \varphi^{-1}\left(y_{2}\right)} f_{p}^{\Omega}(z, \alpha) \\
& =\bigvee_{\substack{x_{1} \in \varphi^{-1}\left(y_{1}\right) \\
x_{2} \in \varphi^{-1}\left(y_{2}\right)}} f_{p}^{\Omega}\left(x_{1} x_{2}, \alpha\right) \geq \bigvee_{\substack{x_{1} \in \varphi^{-1}\left(y_{1}\right) \\
x_{2} \in \varphi^{-1}\left(y_{2}\right)}} \bigwedge\left\{f_{p}^{\Omega}\left(x_{1}, \alpha\right), f_{p}^{\Omega}\left(x_{2}, \alpha\right)\right\} \\
& =\bigwedge\left\{\bigvee_{x_{1} \in \varphi^{-1}\left(y_{1}\right)} f_{p}^{\Omega}\left(x_{1}, \alpha\right), \bigvee_{x_{2} \in \varphi^{-1}\left(y_{2}\right)} f_{p}^{\Omega}\left(x_{2}, \alpha\right)\right\} \\
& =\bigwedge\left\{\varphi\left(f_{p}^{\Omega}\right)\left(y_{1}, \alpha\right), \varphi\left(f_{p}^{\Omega}\right)\left(y_{2}, \alpha\right)\right\}
\end{aligned}
$$

for all $y_{1}, y_{2} \in T$ and $\alpha \in \Omega$. This completes the proof.

\section{Conclusions}

In this paper we have defined the notions of bipolar $\Omega$-fuzzy sub-semigroups in semigroups by using a set $\Omega$. We have described a bipolar $\Omega$-fuzzy sub-semigroup by using a bipolar fuzzy sub-semigroup and vice versa. We have stated how the 
homomorphic images and inverse images of bipolar $\Omega$-fuzzy sub-semigroups become bipolar $\Omega$-fuzzy sub-semigroups. We have discussed normal bipolar $\Omega$-fuzzy subsemigroups, and provided a characterization of a bipolar $\Omega$-fuzzy sub-semigroup. Our future work will focus on studying the bipolar $\Omega$-fuzzy structure with operators of several ideals in semigroups.

\section{REFERENCES}

1. D. Dubois \& H. Prade: Fuzzy sets and systems: Theory and Applications. Academic Press, 1980.

2. K. Hur, Y.B. Jun \& H.S. Kim: Fuzzy sub-semigroups and fuzzy ideals with operators in semigroups. Ann. Fuzzy Math. Inform. 1 (2011), 1-12.

3. C. S. Kim, J. G. Kang \& J. M. Kang: Ideal theory of sub-semigroups based on the bipolar valued fuzzy set theory. Ann. Fuzzy Math. Inform. 2 (2011), 193-206.

4. K. M. Lee: Bipolar-valued fuzzy sets and their operations. Proc. Int. Conf. on Intelligent Technologies, Bangkok, Thailand (2000), 307-312.

5 . _ _ C C Comparison of interval-valued fuzzy sets, intuitionistic fuzzy sets, and bipolarvalued fuzzy sets. J. Fuzzy Logic Intelligent Systems 14 (2004), 125-129.

6. H.-J. Zimmermann: Fuzzy Set Theory and its Applications. Kluwer-Nijhoff Publishing, 1985.

${ }^{a}$ Department of Mathematics, Dongeui University, Busan 614-714, Korea

Email address: mee@deu.ac.kr

b Department of Mathematics Education, Gyeongsang National University, Chinju 660701, KOREA

Email address: jeonggikang@gmail.com 